\title{
EMBEDDINGS OF LOCALLY CONNECTED COMPACTA
}

BY

\author{
GERARD A. VENEMA ${ }^{1}$
}

\begin{abstract}
Let $X$ be a $k$-dimensional compactum and $f: X \rightarrow M^{n}$ a map into a piecewise linear $n$-manifold, $n \geqslant k+3$. The main result of this paper asserts that if $X$ is locally $(2 k-n)$-connected and $f$ is $(2 k-n+1)$-connected, then $f$ is homotopic to a CE equivalence. In particular, every $k$-dimensional, $r$-connected, locally $r$-connected compactum is CE equivalent to a compact subset of $\mathbf{R}^{2 k-r}$ as long as $r \leqslant k-3$.
\end{abstract}

Introduction. Let $X$ be a $k$-dimensional compactum. In this paper we study the problem of finding an embedding of $X$ into Euclidean $n$-space $\mathbf{R}^{n}$. Specifically, we wish to investigate conditions under which we can embed $X$ in $\mathbf{R}^{n}$ when $n$ is less than the classical dimension $2 k+1$.

In case $X$ is a manifold, it has long been known that such improvements are possible. Whitney [Wh] showed that every smooth $k$-dimensional manifold embeds in $\mathbf{R}^{2 k}$. His techniques were later generalized to codimension three embedding theorems for piecewise linear (PL) manifolds by Irwin [Ir] and Hudson [Hd] who showed that if the manifold is $r$-connected, then it is possible to PL embed it in $\mathbf{R}^{2 k-r}$.

The situation for polyhedra is different. In order to accomplish the embedding into a lower-dimensional space it is necessary to identify certain contractible sets to points and this changes the homeomorphism type of the polyhedron. Thus the map produced is not a topological embedding, but rather a simple homotopy equivalence. So the appropriate theory for polyhedra is a theory of embedding up to simple homotopy type and this theory was worked out by Stallings [St]. It follows from his main theorem that every $k$-dimensional, $r$-connected polyhedron is simple homotopy equivalent to a subpolyhedron of $\mathbf{R}^{2 k-r}$.

The purpose of this paper is to prove a theorem like that of Stallings, but for more general compacta rather than polyhedra. In moving from polyhedra to compacta it is necessary to add an additional hypothesis: We must assume that the polyhedra are locally $r$-connected as well as globally $r$-connected. In addition, we must use an appropriate generalization of the (strictly polyhedral) concept of simple homotopy equivalence. We substitute CE equivalence. This is the natural thing to do because

Received by the editors January 3, 1985.

1980 Mathematics Subject Classification. Primary 57Q35, 54F35, 54C10, 57N15; Secondary 57N25. 57 N60.

Key words and phrases. Cell-like set, compactum, CE map, CE equivalence, topological embedding, $r$-connected, locally $r$-connected.

${ }^{1}$ Research partially supported by National Science Foundation grant number MCS- 8301680 . 
every CE map of polyhedra induces a simple homotopy equivalence $[\mathbf{C h}]$ and a simple homotopy equivalence is made up of a sequence of maps, each of which shrinks out cell-like sets of a particular kind.

Before stating our main theorem we give a definition of $\mathrm{CE}$ equivalence.

Definition. A compact space $A$ is said to be a cell-like set if, for some embedding of $A$ into an absolute neighborhood retract (ANR), $A$ has the property that $A$ is contractible in every neighborhood of itself. A map $f: X \rightarrow Y$ of compacta is said to be a $C E$ map if $f$ is onto and $f^{-1}(y)$ is a cell-like subset of $X$ for every $y \in Y$. We say that $f$ is a $C E$ equivalence if there exists a compactum $Z$ and $C E$ maps $g$ : $Z \rightarrow X$ and $h: Z \rightarrow Y$ such that $h=f g$.

THEOREM 1. Suppose $X$ is a locally $r$-connected, $k$-dimensional compactum and $M^{n}$ is a PL n-manifold, $k \leqslant n-3, r \geqslant 2 k-n$. If $f: X \rightarrow M$ is an $(r+1)$-connected map, then $f$ is homotopic to a $C E$ equivalence; i.e., there exist $k$-dimensional compacta $Y$ and $Z$ such that $Y \subset M^{n}$ and $C E$ maps $Z \rightarrow X$ and $Z \rightarrow Y$ such that

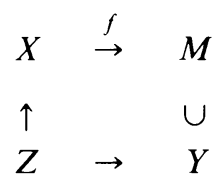

commutes up to homotopy. Furthermore, if $r \leqslant k-2$ then $Y$ is locally $r$-connected.

COROllary. If $X$ is a $k$-dimensional, r-connected, locally r-connected compactum, $r \leqslant k-3$, then $X$ is $C E$ equivalent to a compact, $k$-dimensional, locally $r$-connected subset of $\mathbf{R}^{2 k-r}$.

We note that Theorem 1 has precisely the same connectivity conditions as are found in Stallings' theorem, except that we assume local connectivity as well as global connectivity. The local connectivity is automatic in the case of polyhedra and the example in [D-H] shows that it is a necessary hypothesis in the case of compacta.

There have been other generalizations of [St] to compacta. One such generalization involves the use of shape equivalence instead of $\mathrm{CE}$ equivalence. The main results of that type are contained in $[\mathbf{H}-\mathbf{I}]$. It is interesting to note that the hypotheses used in [H-I] are equivalent (by [Fe1]) to the assumption that $X$ has the shape of an $\mathrm{LC}^{r}$ compactum for precisely the same value of $r$ as is used here.

In addition, Husch [Hs] has proved a metastable range theorem similar to ours but using a condition which is stronger than the $\mathrm{LC}^{r}$ hypothesis. He proves that if $X$ can be expanded as the inverse limit of an inverse sequence of $k$-dimensional polyhedra with $\mathrm{UV}^{r}$ bonding maps, $r \geqslant 2 k-n, k \leqslant n-3$, and $k<(2 / 3)(n-1)$, then any $(r+1)$-connected map $f: X \rightarrow M^{n}$ is homotopic to a CE equivalence. Ferry [Fe1] has shown that if $X$ can be written as an inverse limit of an inverse sequence with $\mathrm{UV}^{r}$ bonding maps, then $X$ is $\mathrm{LC}^{r}$ (while the converse is not true). Thus Husch's theorem follows from Theorem 1. But the proof of Theorem 1 given in this paper is based on Husch's construction. 
The author wishes to express his appreciation to R. J. Daverman, J. Dydak, and L. Husch for helpful conversations regarding the results in this paper. Thanks also to M. Bestvina for the proof of Lemma 2.1.

1. Definitions. A space $X$ is said to be $r$-connected if every map of the $m$-sphere $S^{m}, m \leqslant r$, into $X$ extends to a map of the $(m+1)$-ball $B^{m+1}$ into $X$. We say that $X$ is locally r-connected (abbreviated $\mathrm{LC}^{r}$ ) if for every $x \in X$ and for every neighborhood $U$ of $x$ there exists a neighborhood $V$ of $x$ such that any map of $S^{m}$ into $V$ extends to a map of $B^{m+1}$ into $U, m \leqslant r$. A map $f: X \rightarrow Y$ is said to be an $r$-connected map if $\pi_{i}(M(f), X)=0$ for $i \leqslant r$. Here $M(f)$ denotes the mapping cylinder of $f$.

Let $\varepsilon$ be a positive number. A homotopy $f: X \times[0,1] \rightarrow Y$ is said to be an $\varepsilon$-homotopy if $\operatorname{diam}(f(\{x\} \times[0,1]))<\varepsilon$ for every $x \in X$. A collapse of one polyhedron to another induces a strong deformation retraction in a natural way. The collapse is said to be an $\varepsilon$-collapse if the induced homotopy is an $\varepsilon$-homotopy. A regular neighborhood $N$ of a polyhedron $K$ is said to be an $\varepsilon$-regular neighborhood if $N$ collapses to $K$ via an $\varepsilon$-collapse.

All polyhedra considered in this paper are compact. If $L$ is a polyhedron equipped with a triangulation, then $L^{(r)}$ denotes the $r$-skeleton.

Inverse limits are useful in this paper. If $\left\{X_{i}\right\}$ is a sequence of spaces and $f_{i}$ : $X_{i+1} \rightarrow X_{i}$, we consider $\lim _{\leftarrow}\left\{X_{i}, f_{i}\right\}$ to be the subset of the product space $\Pi X_{i}$ consisting of all sequences $\left(x_{i}\right)$ such that $f_{i}\left(x_{i+1}\right)=x_{i}$.

It is also convenient to use shape theory occasionally to prove that certain sets are cell-like. We use only the most elementary facts which can be found in any book on the subject (see [D-S], for example).

Finally, we mention taming theory. We follow [Ed and Št] and say that a $k$-dimensional compactum $X$ in a PL manifold is tame if for every $\varepsilon>0$ there exists a neighborhood $N$ of $X$ such that $N$ is an $\varepsilon$-regular neighborhood of a $k$-dimensional polyhedron.

2. Neighborhoods. Let $X$ denote a $k$-dimensional, compact, $\mathrm{LC}^{r}$ metric space which is a subset of the interior of a PL $n$-manifold $M$. In this section we will build neighborhoods of $X$ in $M$ which reflect the local connectivity of $X$. Specifically, this means that the neighborhoods should contain small homotopies pushing polyhedra of appropriate dimension into $X$.

Definition. Let $\varepsilon$ be a small positive number. We will call a neighborhood $N$ of $X$ an $(\varepsilon, r)$-deformation neighborhood if for every $(r+1)$-dimensional polyhedron $L$ and every map $f: L \rightarrow N$ there exists an $\varepsilon$-homotopy $f_{t}: L \rightarrow N$ such that $f_{0}=f$ and $f_{1}(L) \subset X$. We say that $N$ is a strong $(\varepsilon, r)$-deformation neighborhood if $N$ has the additional property that if $L_{0}$ is a subpolyhedron of $L$ such that $f\left(L_{0}\right) \subset X$, then we can require that $f_{t}\left|L_{0}=f\right| L_{0}$ for every $t$.

In this section we will prove that, under suitable hypotheses, $\mathrm{LC}^{r}$ compacta have arbitrarily small strong $(\varepsilon, r)$-deformation neighborhoods. The converse is also true: if $X$ has strong $(\varepsilon, r)$-deformation neighborhoods for arbitrarily small $\varepsilon$, then $X$ is 
LC ${ }^{r}$. This is because a map of $S^{m}$ into a small subset of $X$ extends to a map of $B^{m+1}$ into a small subset of $M$. If $m \leqslant r$, a strong $(\varepsilon, r)$-deformation neighborhood can then be used to push the image of $B^{m+1}$ into $X$.

It seems simplest to begin by proving the existence of (weak) $(\varepsilon, r)$-deformation neighborhoods. The proof of the following lemma was communicated to the author by $\mathrm{M}$. Bestvina.

LEMMA 2.1. Suppose $X$ is a $k$-dimensional compactum which is tamely embedded in the PL n-manifold $M$. If $X$ is $L C^{r}, r \leqslant k-2, k+r+3 \leqslant n$, then for every $\varepsilon>0$ there is an $(\varepsilon, r)$-deformation neighborhood $N$ of $X$ in $M$ such that $N$ is an $\varepsilon$-regular neighborhood of a k-dimensional polyhedron $K$.

Proof. The proof is by induction on $r$ and begins with the case $r=-1$ (in which case there is something to prove even though there is no restriction on $X$ ).

Choose $\delta>0$ such that for any point $x \in N_{\delta}(X)$ there exists a path of diameter $<\varepsilon / 5$ from $x$ to a point of $X$. Let $N_{1}$ be a neighborhood of $X$ such that $N_{1}$ is an $(\varepsilon / 5)$-regular neighborhood of a $k$-dimensional polyhedron $K_{1}$ and $N_{1} \subset N_{\delta}(X)$. Take a triangulation of $K_{1}$ having mesh less than $\varepsilon / 5$.

For each vertex $v$ of $K_{1}$, choose an $(\varepsilon / 5)$-arc $B_{v}$, from $v$ to a point of $X$. We may assume that $B_{v} \cap K_{1}=\{v\}$. (If the other end of $B_{v}$, lies in $K_{1} \cap X$, just move $K_{1}$ slightly.) Keep the endpoints of $B_{v}$, fixed and push the rest of $B_{v}$ out of $N_{1}$ using the natural $(\varepsilon / 5)$-product structure on $N_{1}-K_{1} \cong \partial N_{1} \times[0,1)$. The new arc, $C_{l}$, has diameter $<3 \varepsilon / 5$. Now add a small $(\varepsilon / 5)$-regular neighborhood of each arc $C_{v}$, to $N_{1}$. We take $N$ to be $N_{1}$ plus the neighborhoods of the arcs and take $K$ to be $K_{1}$ plus the arcs $C_{v^{\prime}}$ plus the shadows of $C_{v} \cap \partial N_{1}$ under the collapse of $N_{1}$ to $K_{1}$.

Pick a point $p \in N$. We must show that there is an $\varepsilon$-homotopy of $p$ in $N$ which pushes $p$ into $X$. First homotope $p$ into $K$ with an $(\varepsilon / 5)$-homotopy. In case this pushes $p$ into $K_{1}$, continue to push $p$ along $K_{1}$ to the nearest vertex. The other case is that the first homotopy pushes $p$ into one of the arcs $C_{v}$. In either case we get $p$ into one of the $C_{l}$, with a $(2 \varepsilon / 5)$-homotopy. The homotopy is completed by moving along $C_{v}$, into $X$.

Now suppose that $r \geqslant 0$ and that $X$ has $(\varepsilon, r-1)$-deformation neighborhoods for arbitrarily small $\varepsilon$.

Let $\varepsilon>0$ be given. Choose $\delta_{1}>0$ such that any $\delta_{1}$-map of $S^{r+1}$ into the $\delta_{1}$-neighborhood of $X$ extends to an $(\varepsilon / 8)$-map of $B^{r+2}$ into $M$. (The existence of $\delta_{1}$ follows from the fact that $M$ is $\mathrm{LC}^{r+1}$.) Next we use the fact that $X$ is $\mathrm{LC}^{r}$ to choose $\delta>0$ such that $\delta<\delta_{1} / 2$ and if $L$ is any $(r+1)$-complex and $f: L^{(r)} \rightarrow X$ such that $\operatorname{diam}[f(\partial \Delta)]<\delta$ for every $(r+1)$-simplex $\Delta$ in $L$, then $f$ extends to a map $f^{\prime}: L \rightarrow X$ such that $\operatorname{diam}\left[f^{\prime}(\Delta)\right]<\delta_{1} / 2$ for every simplex $\Delta$ in $L$.

Now apply the inductive hypothesis to get a $(\delta / 3, r-1)$-deformation neighborhood $N_{1}$ of $X$ such that $N_{1}$ collapses to a $k$-dimensional polyhedron $K_{1}$ via an $(\varepsilon / 8)$-collapse. Triangulate $K_{1}$ with a triangulation of mesh less than $\delta / 3$ and let $K_{1}^{(r+1)}$ denote the $(r+1)$-skeleton of $K_{1}$.

We claim that there exists an ( $\varepsilon / 8)$-homotopy of $K_{1}^{(r+1)}$ into $X$ which keeps $K_{1}^{(r)}$ in $N_{1}$. By the choice of $N_{1}$, there exists a $(\delta / 3)$-homotopy $h_{t}: K_{1}^{(r)} \rightarrow N_{1}$ such that $h_{0}=$ inclusion and $h_{1}\left(K_{1}^{(r)}\right) \subset X$. Extend $h_{0}$ via the inclusion to all of $K_{1}^{(r+1)}$. For 
each $(r+1)$-simplex $\Delta \subset K_{1}^{(r+1)}, h_{1}(\partial \Delta) \subset X$ and $\operatorname{diam}\left[h_{1}(\partial \Delta)\right]<\delta$. So the choice of $\delta$ allows us to extend $h_{1}$ to $K_{1}^{(r+1)}$ in such a way that $h_{1}\left(K_{1}^{(r+1)}\right) \subseteq X$ and $\operatorname{diam}\left[h_{1}(\Delta)\right]<\delta_{1} / 2$ for each $\Delta \subseteq K_{1}^{(r+1)}$. Then for each $(r+1)$-simplex $\Delta \subseteq K_{1}^{(r+1)}$, $h_{0}(\Delta) \cup h_{1}(\Delta) \cup\left\{h_{t}(\partial \Delta) \mid 0 \leqslant t \leqslant 1\right\}$ has diameter less than $\delta_{1}$. The choice of $\delta_{1}$ now allows us to extend $h_{t}$ to the $(\varepsilon / 8)$-homotopy whose existence was claimed above.

We now have a map $h: K_{1}^{(r+1)} \times[0,1] \rightarrow M$ such that $h(x, 0)=x$ for all $x \in K_{1}^{(r+1)}, h\left(K_{1}^{(r+1)} \times\{1\}\right) \subseteq X, h\left(K_{1}^{(r)} \times[0,1]\right) \subseteq N_{1}$, and $\operatorname{diam}[h(\Delta \times[0,1])]$ $<\varepsilon / 8$ for every $(r+1)$-simplex $\Delta \subset K_{1}^{(r+1)}$. For some small positive number $\gamma$, make $h \mid K_{1}^{(r+1)} \times[0,1-\gamma]$ PL and put it in general position with respect to $K_{1}$. The condition $k+r+3 \leqslant n$ then implies that $h\left(K_{1}^{(r+1)} \times[0,1-\gamma]\right)$ intersects $K_{1}$ only along $h\left(K_{1}^{(r+1)} \times\{0\}\right)=K_{1}^{(r+1)}$. We may also assume that $\gamma$ and the PL approximation were chosen carefully enough so that

$$
h\left(K_{1}^{(r+1)} \times\{0,1-\gamma\}\right) \cup h\left(K_{1}^{(r)} \times[0,1-\gamma]\right) \subset N_{1} .
$$

Push the rest of $h\left(K_{1}^{(r+1)} \times[0,1-\gamma]\right)$ out of $N_{1}$, keeping $h\left(K_{1}^{(r+1)} \times\{0,1-\gamma\}\right)$ $\cup h\left(K_{1}^{(r)} \times[0,1-\gamma]\right)$ fixed. Then $h\left(K_{1}^{(r+1)} \times[0,1-\gamma]\right)$ consists of the part lying in $N_{1}$ together with some $(r+2)$-cells poking out of $N_{1}$. Each of these $(r+2)$-cells has diameter less than $3 \varepsilon / 8$. We take $N$ to be $N_{1}$ plus $(\varepsilon / 8)$-regular neighborhoods of the $(r+2)$-cells and take $K$ to be $K_{1}$ plus the $(r+2)$-cells plus shadows of the $(r+2)$-cells in the product structure of $N_{1}-K_{1}$.

We must now show that $N$ satisfies the conclusion of the lemma. Let $L$ be an $(r+1)$-dimensional polyhedron and $f: L \rightarrow N$. We construct the homotopy of $f(L)$ into $X$ in several stages. First, homotope $f(L)$ out of the $(r+2)$-handles and into $N_{1}$. Next, push $f(L)$ down the product structure of $N_{1}-K_{1}$ into $K_{1}$. Then push along $K_{1}$ into $K_{1}^{(r+1)}$. From there on, use the homotopy $h_{t}$. The second and third of those homotopies are $(\varepsilon / 8)$-homotopies and the first and last are $(3 \varepsilon / 8)$ homotopies, so the entire homotopy is an $\varepsilon$-homotopy.

Lemma 2.2. Suppose $X$ is a compact $L C^{0}$ subset of the PL manifold $M$. Then for every $\varepsilon>0$ there exists a $\delta>0$ such that every $(\delta, 0)$-deformation neighborhood of $X$ is a strong $(\varepsilon, 0)$-deformation neighborhood of $X$.

Proof. Let $\varepsilon>0$ be given. Choose $\delta>0$ such that $3 \delta \leqslant \varepsilon / 6$ and such that if $x$, $y \in X$ and $d(x, y)<3 \delta$, then $x$ and $y$ can be joined by an arc in $X$ of diameter less than $\varepsilon / 6$.

Now suppose that $N$ is a $(\delta, 0)$-deformation neighborhood of $X$ in $M$. Let $L$ be a 1-dimensional polyhedron, $L_{0}$ a subpolyhedron of $L$ and $f: L \rightarrow N$ a map such that $f\left(L_{0}\right) \subseteq X$. Choose a triangulation of $L$ of such small mesh that $\operatorname{diam}[f(\Delta)]<\delta$ for every simplex $\Delta$ in $L$. For each vertex $v$ of $L-L_{0}$, there is a $\delta$-homotopy of $f(v)$ into $X$. Extend those homotopies via the identity to $f\left(L_{0}\right)$ and then to a $\delta$-homotopy of all of $f(L)$ in $N$.

Now if $\Delta$ is a 1 -simplex in $L$, we have $\delta$-homotoped $f \mid \Delta$ to a map $f^{\prime}: \Delta \rightarrow N$ such that $f^{\prime}(\partial \Delta) \subseteq X$ and $\operatorname{diam}\left[f^{\prime}(\Delta)\right]<3 \delta \leqslant \varepsilon / 6$. Thus the proof of the lemma will be complete if we can show that $f^{\prime}$ is $(5 \varepsilon / 6)$-homotopic, rel $\partial \Delta$, to a map of $\Delta$ into $X$. To do so we use a base point trick just like that in [L-V, Lemma 1]. 
By the choice of $\delta$, we can extend $f^{\prime} \mid \partial \Delta$ to a map $f^{\prime \prime}: \Delta \rightarrow X$ such that $\operatorname{diam}\left[f^{\prime \prime}(\Delta)\right]<\varepsilon / 6$. Let $\Omega$ be the loop made up of $f^{\prime}(\Delta)$ together with $f^{\prime \prime}(\Delta)$ with reverse orientation. We want to show that $\Omega$ is homotopic, rel base point, to a loop $\Omega^{\prime}$ in $X$. If so, then $f^{\prime}(\Delta)$ is homotopic (rel base point) to the path $f^{\prime \prime}(\Delta)$ followed by $\Omega^{\prime}$ which lies entirely in $X$ and so we are finished.

Take a triangulation of $N$ of mesh less than $\delta$ and let $K$ denote the 1-skeleton of the triangulation. By hypothesis, there exists a $\delta$-homotopy $h_{t}: K \rightarrow N$ such that $h_{0}=$ inclusion and $h_{1}(K) \subset X$. By moving $f^{\prime \prime}$ slightly (if necessary) we may assume that there is a point $y \in K$ such that $h_{1}(y) \in f^{\prime \prime}(\Delta)$. This may increase the size of $f^{\prime \prime}(\Delta)$, but only by $\varepsilon / 6$. We will first use $y$ as a base point. Let $a$ be the path from $y$ to $h_{1}(y)$ traced out by the homotopy $h_{t} \mid\{y\}$. Notice that there is a base-point-fixing $2 \delta$-homotopy of the loop $a \Omega a^{-1}$ to a loop of the form $a \Omega^{\prime} a^{-1}$ where $\Omega^{\prime}$ is a loop in $X$. (This homotopy consists of a push into the 1-skeleton $K$, followed by the homotopy $h_{t}$.) But now using $h_{1}(y)$ as a base point, we see that $\Omega \simeq a^{-1} a \Omega a^{-1} a \simeq a^{-1} a \Omega^{\prime} a^{-1} a \simeq \Omega^{\prime}$ where " $\simeq$ " denotes "is homotopic to" and all the homotopies are rel the base point $h_{1}(y)$. To complete the proof we need only compute the size of the homotopies involved. The entire image of the homotopy has

$$
\begin{aligned}
\operatorname{diameter} & \leqslant \operatorname{diam}[\Omega]+\operatorname{diam}[a]+4 \delta+\operatorname{diam}[a] \\
& \leqslant\left(\operatorname{diam}\left[f^{\prime}(\Delta)\right]+\operatorname{diam}\left[f^{\prime \prime}(\Delta)\right]+\varepsilon / 6\right)+\delta+4 \delta+\delta \\
& \leqslant 3 \delta+\varepsilon / 6+\varepsilon / 6+6 \delta<5 \varepsilon / 6 .
\end{aligned}
$$

The following is the main result of this section.

Proposition 2.3. Suppose $X$ is a k-dimensional compactum which is tamely embedded in the PL n-manifold $M$. If $X$ is $L C^{r}, r \leqslant k-2$, and $k+r+3 \leqslant n$, then for every $\varepsilon>0$ there is a strong $(\varepsilon, r)$-deformation neighborhood $N$ of $X$ in $M$ such that $N$ is an $\varepsilon$-regular neighborhood of a k-dimensional polyhedron $K$.

REMARK. The hypothesis $r+k+3 \leqslant n$ could be weakened considerably. But doing so would require extra work and we do not have any need for a stronger result in this paper.

Proof. The proof is by induction on $r$. The case $r=-1$ has already been done because there is no difference between an $(\varepsilon,-1)$-deformation neighborhood and a strong $(\varepsilon,-1)$-deformation neighborhood. The case $r=0$ is covered by Lemmas 2.1 and 2.2. So we may assume inductively that $r \geqslant 1$ and that for every $\varepsilon>0$ there is a $\delta>0$ such that every $(\delta, r-1)$-deformation neighborhood of $X$ is a strong $(\varepsilon$, $r-1)$-deformation neighborhood of $X$.

Let $\varepsilon>0$ be given. Use the fact that $X$ is $\mathrm{LC}^{r}$ to choose $\delta_{1}>0$ such that any map $g: S^{r} \rightarrow X$ with $\operatorname{diam}\left[g\left(S^{r}\right)\right]<3 \delta_{1}$ extends to a map $G: B^{r+1} \rightarrow X$ such that $\operatorname{diam}\left[G\left(B^{r+1}\right)\right]<\varepsilon / 5$. By the inductive hypothesis there exists $\delta>0$ such that any $(\delta, r-1)$-deformation neighborhood of $X$ is a strong $\left(\delta_{1}, r-1\right)$-deformation neighborhood. We may also assume that $\delta \leqslant \delta_{1}$ and $3 \delta_{1} \leqslant \varepsilon / 5$.

By Lemma 2.1 there exists a neighborhood $N$ of $X$ such that $N$ is a $(\delta, r)$-deformation neighborhood and such that $N$ is a $\delta$-regular neighborhood of a $k$-dimensional polyhedron $K$. We will show that such an $N$ is a strong $(\varepsilon, r)$-deformation neighborhood. 
Let $L$ be an $(r+1)$-dimensional polyhedron, $L_{0}$ a subpolyhedron of $L$ and $f$ : $L \rightarrow N$ a map such that $f\left(L_{0}\right) \subset X$. We must produce an $\varepsilon$-homotopy $f_{t}: L \rightarrow N$ such that $f_{0}=f, f_{1}(L) \subset X$, and $f_{t}\left|L_{0}=f\right| L_{0}$ for every $t$. We may assume that $L_{0}$ is $r$-dimensional, because otherwise we could restrict our attention to $\operatorname{Clos}\left(L-L_{0}\right)$, construct such a homotopy of $\operatorname{Clos}\left(L-L_{0}\right)$ which keeps $L_{0} \cap \operatorname{Clos}\left(L-L_{0}\right)$ fixed and then extend it via $f$ to all of $L=\operatorname{Clos}\left(L-L_{0}\right) \cup L_{0}$.

Pick a triangulation for $L$ such that if $\Delta$ is a simplex in $L$, then $\operatorname{diam}[f(\Delta)]<\delta$ and $L_{0}$ is a subcomplex of this triangulation. By the choice of $N$, there is a $\delta_{1}$-homotopy $h_{t}: L \rightarrow N$ such that $h_{0}=f, h_{t}\left|L_{0}=f\right| L_{0}$ for each $t$, and $h_{1}\left(L^{(r)}\right) \subset$ $X$. For each $(r+1)$-simplex $\Delta$ of $L$, we have that $h_{1}(\partial \Delta) \subset X$ and $\operatorname{diam}\left[h_{1}(\Delta)\right]<$ $3 \delta_{1}$. Just as in the proof of Lemma 2.2 , we will be finished if we can show that there is a $(4 \varepsilon / 5)$-homotopy of $h_{1}(\Delta)$ into $X$ which keeps $h_{1}(\partial \Delta)$ fixed.

So consider one such $\Delta$. By the choice of $\delta_{1}$, there is a map $h^{\prime}: \Delta \rightarrow X$ which extends $h_{1} \mid \partial \Delta$ and such that $\operatorname{diam}\left[h^{\prime}(\Delta)\right]<\varepsilon / 5$. Consider the singular $(r+1)$-sphere $S=h_{1}(\Delta) \cup h^{\prime}(\Delta)$. There is a $\delta$-homotopy of $S$ to a singular $(r+1)$-sphere $S^{\prime} \subset X$. Pick a base point $x \in S \cap X$. The path $p$ followed by $x$ during the homotopy from $S$ to $S^{\prime}$ is a $\delta$-path beginning and ending on $X$. By induction, there exists a $\delta_{1}$-homotopy of $p$ into $X$ which keeps the ends of $p$ fixed. Let $p^{\prime}$ be the new path in $X$. Notice that $\operatorname{diam}\left[p^{\prime}\right] \leqslant \delta+2 \delta_{1}$ and that $S$ is homotopic (rel base point) to the singular $(r+1)$-sphere $S^{\prime \prime} \subset X$ which is $S^{\prime}$ acted on by the path $p^{\prime}$. Therefore $h_{1} \mid \Delta$ is homotopic, rel $\partial \Delta$, to a map of $\Delta$ onto $S^{\prime \prime} \cup h^{\prime}(\Delta)$.

The size of that homotopy is no greater than $\operatorname{diam}[S]+2 \delta+2 \delta_{1}$. But $\operatorname{diam}[S] \leqslant$ $3 \delta_{1}+\varepsilon / 5$, so the size of the homotopy is no more than $3 \delta_{1}+\varepsilon / 5+2 \delta+2 \delta_{1}<$ $4 \varepsilon / 5$.

Proposition 2.3 seems like the simplest, most natural way to state the properties of the neighborhoods we have constructed. But in the proof of Theorem 1 we will actually need the following, more technical, statement.

Proposition 2.4. Let $X$ be an $L C^{r}$ compactum in the PL $n$-manifold $M$ and let $\varepsilon$ be a positive number. Suppose $N_{1}$ is a strong $(\varepsilon, r)$-deformation neighborhood of $X, \rho$ : $N_{1} \rightarrow K_{1}$ is an $\varepsilon$-retraction onto a k-dimensional spine $K_{1}, N_{0} \subseteq N_{1}$ is a second $(\varepsilon, r)$-deformation neighborhood of $X, L$ is an $(r+1)$-dimensional polyhedron and $L_{0}$ is a subpolyhedron of $L$. Then if $f_{0}: L_{0} \rightarrow N_{0}$ is a map such that $\rho f_{0}$ extends to a map $f: L \rightarrow K_{1}$, then there is an extension $f^{\prime}: L \rightarrow N_{0}$ of $f_{0}$ such that $f$ and $\rho f^{\prime}$ are $5 \varepsilon$-homotopic, rel $L_{0}$, in $K_{1}$.

Proof. We will show that there is a $3 \varepsilon$-homotopy $g_{t}: L \rightarrow N_{1}$ such that $g_{0}=f$, $g_{1}(L) \subset N_{0}, g_{1} \mid L_{0}=f_{0}$ and $\rho\left(g_{t}(x)\right)=f(x)$ for every $x \in L_{0}$ and for every $t$. Once such a homotopy has been constructed, we simply take $f^{\prime}=g_{1}$ and notice that the homotopy $\rho g_{t}$ is a $5 \varepsilon$-homotopy from $\rho g_{0}=f$ to $\rho g_{1}=\rho f^{\prime}$.

To construct $g_{t}$ we proceed as follows. Pick a barycentric subdivision of $L$ having very small mesh. Let $A$ denote the simplicial neighborhood of $L_{0}$ in this triangulation, $C=\operatorname{Clos}(L-A)$ and $B=A \cap C$. Since $N_{0}$ is an $(\varepsilon, r)$-deformation neighborhood, there exists an $\varepsilon$-homotopy of $f\left(L_{0}\right)$ in $N_{0}$ which pushes $f\left(L_{0}\right)$ into $X$. By 
spreading that homotopy out over the neighborhood $A$, we construct a map $F$ : $L \times[0,1] \rightarrow N_{1}$ such that $F(x, 0)=f(x)$ for all $x \in L, F(x, 1)=f_{0}(x)$ for all $x \in L_{0}, F(x, 1) \in N_{0}$ for all $x \in A, F(x, 1) \in X$ for all $x \in B, \rho(F(x, t))=f(x)$ for all $x \in L_{0}$ and all $t$, and $\operatorname{diam}[F(\{x\} \times[0,1])]<2 \varepsilon$ for all $t$. Now there exists an $\varepsilon$-homotopy which pushes $F(C \times\{1\})$ into $X$ keeping $F(B \times\{1\})$ fixed because of the fact that $N_{1}$ is a strong $(\varepsilon, r)$-deformation neighborhood. The homotopy $g_{t}$ is the homotopy obtained by doing the two homotopies mentioned above in succession.

3. Inverse limits and CE limit maps. In this section we give conditions under which a sequence of CE maps into neighborhoods of $X$ converges to a CE map onto $X$. We first show that if $X$ is defined as the intersection of a sequence of neighborhoods with sufficiently small collapses onto their spines, then there is a natural CE map from the inverse limit of the spines onto $X$. Then we construct a CE map from an inverse limit onto $X$ as the limit of a sequence of level maps into that inverse sequence.

The following notation is assumed throughout this section: $X$ is a compactum in the interior of the PL $n$-manifold $M,\left\{N_{i}\right\}$ is a sequence of neighborhoods of $X$, $N_{i+1} \subset$ Int $N_{i}$ for each $i, X=\bigcap N_{i}, N_{i}$ collapses to a compact polyhedron $X_{i}$ via an $\varepsilon_{i}$-collapse $\xi_{i}$, and $\left\{\varepsilon_{i}\right\}$ is a sequence of positive numbers which converges to 0 . Let $\rho_{i}: N_{i} \rightarrow X_{i}$ denote the $\varepsilon_{i}$-retraction induced by $\xi_{i}$ and let $f_{i}: X_{i+1} \rightarrow X_{i}$ be the restriction of $\rho_{i}$. We will refer to the sequence $\left\{N_{i}, X_{i}\right\}$ as a defining sequence for $X$. Notice that the defining sequence $\left\{N_{i}, X_{i}\right\}$ implicitly defines the maps $f_{i}$ and the numbers $\varepsilon_{i}$.

Lemma 3.1. Suppose $X$ is a compact subset of the PL n-manifold $M$. If $\left\{N_{i}, X_{i}\right\}$ is a defining sequence for $X$ such that $\sum \varepsilon_{i}<\infty$, then there is a natural $C E$ map $G$ : $\lim _{\leftarrow}\left\{X_{i}, f_{i}\right\} \rightarrow X$.

REMARK. It is possible to make $G$ a homeomorphism by putting further restrictions on the defining sequence. To do so, the $\varepsilon_{i}$ would have to be chosen inductively and would depend on the preceding retractions. A proof of this stronger lemma would follow the lines of the proofs of Theorems 1 and 2 in [Br].

Proof of Lemma 3.1. Let $\left(x_{i}\right) \in \lim \left\{X_{i}\right\}$. The facts that $d\left(x_{i}, x_{i+1}\right)<\varepsilon_{i}$ and $\sum \varepsilon_{i}<\infty$ imply that the sequence $\left\{x_{i}\right\}$ forms a Cauchy sequence when considered as a sequence of points in $M$. Therefore there is a unique point $y$ such that $x_{i} \rightarrow y$ as $i \rightarrow \infty$. Notice that $y$ must be a point in $X$, so we can define $G\left(\left(x_{i}\right)\right)=y$. It is clear that $G$ is continuous, so the proof will be complete if we show that $G^{-1}(x)$ is a nonempty cell-like set for each $x \in X$.

Fix $x \in X$. Define $C_{i}=\left\{y \in N_{i} \mid d(y, x)<\sum_{k \geqslant i} \varepsilon_{k}\right\}$ and $D_{i}=C_{i} \cap X_{i}$. It is clear that $\cap C_{i}=\{x\}$; i.e., if $\beta_{i}: C_{i+1} \rightarrow C_{i}$ denotes the inclusion map, then $\{x\}=$ $\lim _{\leftarrow}\left\{C_{i}, \beta_{i}\right\}$. Pick $y \in D_{i+1}$; then $y \in X_{i+1}$ and $d(x, y)<\sum_{k \geqslant i+1} \varepsilon_{k}$, so $d\left(x, f_{i}(y)\right)$ $<\sum_{k \geqslant i} \varepsilon_{k}$. Thus $f_{i}\left(D_{i+1}\right) \subset D_{i}$. We finish the proof by showing the following: $G^{-1}(x)=\lim _{\leftarrow}\left\{D_{i}, f_{i} \mid D_{i+1}\right\}$ and $\operatorname{Sh}\left(\lim _{\leftarrow}\left\{D_{i}, f_{i} \mid D_{i+1}\right\}\right)=\operatorname{Sh}\left(\lim _{\leftarrow}\left\{C_{i}, \beta_{i}\right\}\right)$. This will 
complete the proof because then $G^{-1}(x)$ is nonempty (it is the inverse limit of an inverse sequence of nonempty compacta) and it is cell-like because it has the shape of a point.

Consider the diagram:

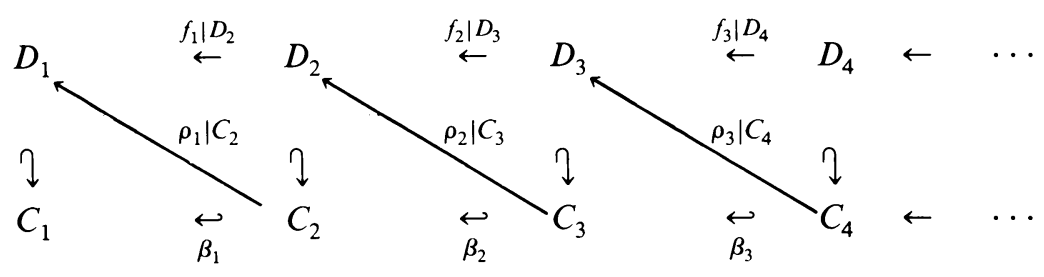

There is an $\varepsilon_{i}$-homotopy from $\beta_{i}$ to $\rho_{i} \mid C_{i+1}$. That homotopy stays in the $\varepsilon_{i}$-neighborhood of $C_{i+1}$, and thus stays in $C_{i}$. Therefore each of the lower triangles in the diagram commutes up to homotopy while each of the upper triangles commutes exactly. Thus we have a shape equivalence from $\lim \left\{D_{i}, f_{i} \mid D_{i+1}\right\}$ to $\lim \left\{C_{i}, \beta_{i}\right\}$.

We now show that $G^{-1}(x)=\lim _{\leftarrow}\left\{D_{i}, f_{i} \mid \overleftarrow{D_{i+1}}\right\}$. Pick $\left(x_{i}\right) \in \overleftarrow{G}^{-1}(x)$. Then $d\left(x_{i}, x_{i+1}\right)<\varepsilon_{i}$ and $x_{i} \rightarrow x$. Thus $d\left(x_{i}, x\right) \leqslant \sum_{k \geqslant i} \varepsilon_{k}$ and $x_{i} \in D_{i}$. On the other hand, if $\left(x_{i}\right) \in \lim _{\leftarrow}\left\{D_{i}\right\}$, then $d\left(x_{i}, x\right) \leqslant \sum_{k \geqslant i} \varepsilon_{k}$. So $x_{i} \rightarrow x$ as $i \rightarrow \infty$ and $\left(x_{i}\right) \in$ $G^{-1}(x)$.

REMARK. Since every PL manifold has a metric in which closed balls are subpolyhedra, we may assume that the $D_{i}$ in the proof above are subpolyhedra.

The following proposition is similar to Proposition 15 of [Hs]. The main difference is that we do not assume that the bonding maps in the inverse sequences are onto, but use Lemma 3.1 instead.

Proposition 3.2. Let $X$ be a compact subset of the PL n-manifold $M$ and let $\left\{N_{i}, X_{i}\right\}$ be a defining sequence for $X$ such that $\sum \varepsilon_{i}<\infty$. Suppose $\left\{Z_{i}, g_{i}\right\}$ is an inverse sequence of compact polyhedra and that for each $i$ there is a PLCE map $\lambda_{i}$ : $Z_{i} \rightarrow X_{i}$. If $f_{i} \lambda_{i+1}$ is $\alpha_{i}$-homotopic to $\lambda_{i} g_{i}$ for each $i$ and $\sum \alpha_{i}<\infty$, then $\Lambda$ : $\lim _{\leftarrow}\left\{Z_{i}, g_{i}\right\} \rightarrow X$ defined by $\Lambda\left(\left(z_{i}\right)\right)=\lim _{i \rightarrow \infty} \lambda_{i}\left(z_{i}\right)$ is a CE map.

Proof. Let $Z=\lim _{\leftarrow}\left\{Z_{i}, g_{i}\right\}$. Define $\Lambda_{j}: Z \rightarrow N_{1}$ by $\Lambda_{j}\left(\left(z_{i}\right)\right)=\lambda_{j}\left(z_{j}\right)$. Then

$$
\begin{gathered}
d\left(\Lambda_{j+1}\left(\left(z_{i}\right)\right), \Lambda_{j}\left(\left(z_{i}\right)\right)\right)=d\left(\lambda_{j+1}\left(z_{j+1}\right), \lambda_{j}\left(z_{j}\right)\right)=d\left(\lambda_{j+1}\left(z_{j+1}\right), \lambda_{j} g_{j}\left(z_{j+1}\right)\right) \\
\leqslant d\left(\lambda_{j+1}\left(z_{j+1}\right), f_{j} \lambda_{j+1}\left(z_{j+1}\right)\right)+d\left(f_{j} \lambda_{j+1}\left(z_{j+1}\right), \lambda_{j} g_{j}\left(z_{j+1}\right)\right) \\
\leqslant \varepsilon_{j}+\alpha_{j} .
\end{gathered}
$$

Thus $\left\{\Lambda_{j}\right\}$ forms a Cauchy sequence of maps and therefore converges to a continuous map $\Lambda: Z \rightarrow X$.

Fix $x \in X$. To complete the proof we must show that $\Lambda^{-1}(x)$ is a nonempty cell-like subset of $Z$. Define $D_{i}=\left\{y \in X_{i} \mid d(x, y) \leqslant \sum_{k \geqslant i}\left(\varepsilon_{k}+\alpha_{k}\right)\right\}$ and $E_{i}=$ $\lambda_{i}^{-1}\left(D_{i}\right)$. Notice that $f_{i}\left(D_{i+1}\right) \subset D_{i}$. It is also true that $g_{i}\left(E_{i+1}\right) \subset E_{i}$. To see this, choose $w \in E_{i+1}$. We must show that $g_{i}(w) \in E_{i}=\lambda_{i}^{-1}\left(D_{i}\right)$ which is equivalent to 
showing that $\lambda_{i} g_{i}(w) \in D_{i}$.

$$
\begin{aligned}
d\left(\lambda_{i} g_{i}(w), x\right) & \leqslant d\left(\lambda_{i} g_{i}(w), f_{i} \lambda_{i+1}(w)\right)+d\left(f_{i} \lambda_{i+1}(w), x\right) \\
& \leqslant \alpha_{i}+\left[\varepsilon_{i}+d\left(\lambda_{i+1}(w), x\right)\right] \\
& \leqslant \alpha_{i}+\varepsilon_{i}+\sum_{k \geqslant i+1}\left(\varepsilon_{k}+\alpha_{k}\right)=\sum_{k \geqslant i}\left(\alpha_{k}+\varepsilon_{k}\right) .
\end{aligned}
$$

Thus $\lambda_{i} g_{i}(w) \in E_{i}$.

Let $G$ be the map defined in the proof of Lemma 3.1. We make the following three claims.

(i) $\Lambda^{-1}(x)=\lim _{\leftarrow}\left\{E_{i}, g_{i} \mid E_{i+1}\right\}$.

(ii) $\operatorname{Sh}\left(\lim _{\leftarrow}\left\{E_{i}, g_{i} \mid E_{i+1}\right\}\right)=\operatorname{Sh}\left(\lim _{\leftarrow}\left\{D_{i}, f_{i} \mid D_{i+1}\right\}\right)$.

(iii) $\lim _{\leftarrow}^{\leftarrow}\left\{D_{i}, f_{i} \mid D_{i+1}\right\}=G^{-1}(x)$.

Once claims (i)-(iii) are verified, the proof is complete.

Let $w \in \Lambda^{-1}(x)$. Then $w=\left(w_{i}\right)$ and $\lambda_{i}\left(w_{i}\right) \rightarrow x$ as $i \rightarrow \infty$. But

$$
d\left(\lambda_{i+1}\left(w_{i+1}\right), \lambda_{i}\left(w_{i}\right)\right) \leqslant \alpha_{i}+\varepsilon_{i}
$$

(by the calculation at the beginning of this proof) and so $w_{i} \in E_{i}$. On the other hand, if $\left(w_{i}\right) \in \lim _{\leftarrow}\left\{E_{i}\right\}$, then $d\left(\lambda_{i}\left(w_{i}\right), x\right) \leqslant \varepsilon_{i}+\alpha_{i}$, so $\lambda_{i}\left(w_{i}\right) \rightarrow x$ and $\left(w_{i}\right) \in \Lambda^{-1}(x)$. Thus claim (i) is verified.

The following diagram is homotopy commutative for each $i$.

$$
\begin{array}{lll}
D_{i} & f_{i} \mid D_{i+1} & D_{i+1} \\
\uparrow \lambda_{i} \mid E_{i} & & \uparrow \lambda_{i+1} \mid E_{i+1} \\
E_{i} & \underset{g_{l} \mid E_{i+1}}{\leftarrow} & E_{i+1}
\end{array}
$$

Furthermore, $\lambda_{i}\left|E_{i}=\lambda_{i}\right| \lambda_{i}^{-1}\left(D_{i}\right)$ is a homotopy equivalence [La, Theorem 1.2]. Therefore $\left\{\lambda_{i} \mid E_{i}\right\}$ induces a shape equivalence from $\lim \left\{E_{i}, g_{i} \mid E_{i+1}\right\}$ to $\lim _{\leftarrow}\left\{D_{i}, f_{i} \mid D_{i+1}\right\}$ and claim (ii) is verified.

The proof of claim (iii) is just like the proof of the corresponding claim in the proof of Lemma 3.1. This completes the proof of Proposition 3.2.

4. A technical lemma. Theorem 1 will be proved by constructing an infinite sequence of CE maps and then taking limits as in Proposition 3.2. The lemma in this section is the inductive step in that infinite construction. It is an $\varepsilon$-controlled version of [St] and corresponds to Proposition 6 of [Hs].

LEMma 4.1. Let $X$ be a $k$-dimensional, $L C^{r}$ compactum tamely embedded in $\mathbf{R}^{m}$, $m \geqslant 2 k+3, r \leqslant k-2$, and let $M$ be a PL $n$-manifold, $n \geqslant k+3$ and $n \geqslant 2 k-r$. If $\varepsilon_{1}, \varepsilon_{2}, \alpha_{1}$ and $\alpha_{2}$ are positive numbers and

$$
X_{1} \stackrel{\lambda_{1}}{\leftarrow} Z_{1} \stackrel{\mu_{1}}{\rightarrow} Y_{1}
$$

satisfies

(4.1) $X_{1}$ is a $k$-dimensional polyhedron which is the spine of a strong $\left(\varepsilon_{1}, r\right)$-deformation neighborhood $N_{1}$ of $X$ in $\mathbf{R}^{m}$; 
(4.2) $N_{1}$ retracts to $X_{1}$ via an $\varepsilon_{1}$-retraction $\rho_{1}: N_{1} \rightarrow X_{1}$;

(4.3) $Z_{1}=X_{1} \cup M_{1}$, where $M_{1}$ is $(r+2)$-dimensional;

(4.4) $M_{1} \cap X_{1}$ is $(r+1)$-dimensional and collared in $M_{1}$;

(4.5) $\lambda_{1}: Z_{1} \rightarrow X_{1}$ is a PL CE retraction with $\lambda_{1}\left(M_{1}\right) \subset M_{1} \cap X_{1}$;

(4.6) $Y_{1}$ is a $k$-dimensional subpolyhedron of $M$;

(4.7) $\mu_{1}: Z_{1} \rightarrow Y_{1}$ is a PL CE map;

(4.8) if $A \subset X_{1}$ and $\operatorname{diam}[A]<\varepsilon_{1}$, then $\operatorname{diam}\left[\mu_{1}(A)\right]<\alpha_{1}$;

(4.9) if $A \subset N_{1}$ and $\operatorname{diam}[A]<\varepsilon_{2}$, then $\operatorname{diam}\left[\mu_{1} \rho_{1}(A)\right]<\alpha_{2}$; and

(4.10) $P_{1}$ is a regular neighborhood of $Y_{1}$ and $q: P_{1} \rightarrow Y_{1}$ is a PL $\alpha_{1}$-retraction; then there exists

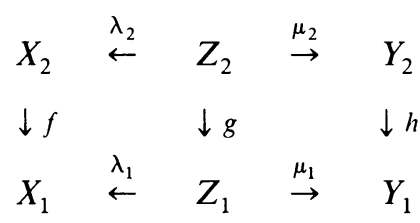

which satisfies

(4.11) $X_{2}$ is a $k$-dimensional polyhedron which is the spine of a strong $\left(\varepsilon_{2}, r\right)$-deformation neighborhood $N_{2}$ of $X$ in $N_{1}$;

(4.12) $f=\rho_{1} \mid X_{2}$

(4.13) $Z_{2}=X_{2} \cup M_{2}$, where $M_{2}$ is $(r+2)$-dimensional;

(4.14) $M_{2} \cap X_{2}$ is $(r+1)$-dimensional and collared in $M_{2}$;

(4.15) $\lambda_{2}: Z_{2} \rightarrow X_{2}$ is a PL CE retraction with $\lambda_{2}\left(M_{2}\right) \subset M_{2} \cap X_{2}$;

(4.16) $Y_{2}$ is a $k$-dimensional polyhedron in $P_{1}$;

(4.17) $h=q \mid Y_{2}$;

(4.18) $\mu_{2}: Z_{2} \rightarrow Y_{2}$ is a PL CE map;

(4.19) $\mu_{2} \mid X_{2}$ is so close to $\mu_{1} \rho_{1} \mid X_{2}$ that if $A \subset X_{2}$ and $\operatorname{diam}[A]<\varepsilon_{2}$, then $\operatorname{diam}\left[\mu_{2}(A)\right]<\alpha_{2}$;

(4.20) $\lambda_{1} g$ is $4(r+1) \varepsilon_{1}$-homotopic to $f \lambda_{2}$ in $K_{1}$; and

(4.21) $\mu_{1} g$ is $8(r+1) \alpha_{1}$-homotopic to $h \mu_{2}$ in $Y_{1}$.

PRoof. We may assume that $\varepsilon_{2} \leqslant \varepsilon_{1}$. Let $N_{2}$ be a strong $\left(\varepsilon_{2}, r\right)$-deformation neighborhood of $X$ in $N_{1}$ which $\varepsilon_{2}$-collapses to a $k$-dimensional polyhedron $X_{2}$. Let $\rho_{2}: N_{2} \rightarrow X_{2}$ be the $\varepsilon_{2}$-retraction induced by the collapse of $N_{2}$ to $X_{2}$ and let $f=\rho_{1} \mid X_{2}$.

We next construct $Z_{2}$. It will be constructed in two steps. First we will lift the attaching map for $M_{1}$ to $X_{2}$ and form $Z^{\prime}$ by attaching $M_{1}$ to $X_{2}$. Then $Z_{2}$ will be formed by using an $\varepsilon$-controlled version of Stallings [St] to attach further mapping cylinders to $Z^{\prime}$. The details follow.

Let $\beta: M_{1} \cap X_{1} \rightarrow X_{1}$ denote the inclusion map. Since $N_{1}$ is an $\left(\varepsilon_{1}, r\right)$-deformation neighborhood, there exists $\beta^{\prime}: M_{1} \cap X_{1} \rightarrow X_{2}$ such that $\beta^{\prime}$ is $2 \varepsilon_{1}$-homotopic to $\beta$ in $N_{1}$. Define $Z^{\prime}=X_{2} \cup_{\beta^{\prime}} M_{1}$. Notice that $f \beta^{\prime}$ is $4 \varepsilon_{1}$-homotopic to $\beta$ in $X_{1}$. Thus there is a map $g: Z^{\prime} \rightarrow Z_{1}$ such that $g \mid X_{2}=f$ and $d(x, g(x))<4 \varepsilon_{1}$ for every $x \in M_{1}$. The map $g$ is just $f$ on $X_{2}$, the identity on $M_{1}$ minus a small collar on 
$M_{1} \cap X_{1}$ and stretches the collar out over the homotopy from $\beta$ to $f \beta^{\prime}$. Furthermore, $\lambda_{1}$ induces a retraction $\lambda^{\prime}: Z^{\prime} \rightarrow X_{2}$ such that $\lambda_{1} g$ and $f \lambda^{\prime}$ are $4 \varepsilon_{1}$-homotopic in $X_{1}$.

Consider $\mu_{1} g: Z^{\prime} \rightarrow Y_{1} \subset P_{1}$. Let $g^{\prime}: Z^{\prime} \rightarrow P_{1}$ be a PL general position map which is $\alpha_{1}$-homotopic to $\mu_{1} g$. Let $\Sigma$ denote the singular set of $g^{\prime}$ and let $M^{\prime}$ denote the mapping cylinder of $g^{\prime} \mid \Sigma: \Sigma \rightarrow g^{\prime}(\Sigma)$. Then $\operatorname{dim} \Sigma \leqslant r$ and $\operatorname{dim} M^{\prime} \leqslant r+1$. We identify $\Sigma$ with $\Sigma \times\{0\} \subset M^{\prime}$ and let $\xi: M^{\prime} \rightarrow g^{\prime}(\Sigma)$ denote the map which collapses out the fibers of the mapping cylinder.

We now wish to extend the inclusion map $\Sigma \subset Z^{\prime}$ to a map of $M^{\prime}$ into $Z^{\prime}$. The $\alpha_{1}$-homotopy from $g^{\prime}$ to $\mu_{1} g$ gives us a map $\tau_{1}: M^{\prime} \rightarrow P_{1}$ such that $\tau_{1}\left|\Sigma=\mu_{1} g\right| \Sigma$, $\tau_{1} \mid g^{\prime}(\Sigma)=$ inclusion and the diameter of the image under $\tau_{1}$ of each fiber of $M^{\prime}$ is less than $\alpha_{1}$. Then $q \tau_{1}: M^{\prime} \rightarrow Y_{1}$ and $q \tau_{1}\left|\Sigma=\mu_{1} g\right| \Sigma$. Notice that the $\alpha_{1}$-homotopy from $\tau_{1}$ to $q \tau_{1}$ can be used to construct an $\alpha_{1}$-homotopy form $q \tau_{1}$ to $\xi$. The homotopy is obviously not rel $\Sigma$, but it does have the property that, when restricted to $\Sigma$, it is just the reverse of the $\alpha_{1}$-homotopy from $g^{\prime}$ to $\mu_{1} g$. Since $\mu_{1}$ is a CE map, there exists a lift $\tau_{2}: M^{\prime} \rightarrow Z_{1}$ such that $\tau_{2}|\Sigma=g| \Sigma$ and such that $\mu_{1} \tau_{2}$ is $\alpha_{1}$-homotopic to $q \tau_{1}$ rel $\Sigma$. We may assume that $\tau_{2}$ is PL. The next step is to lift to $Z^{\prime}$. We define $\tau_{3}: M^{\prime} \rightarrow Z^{\prime}$ as follows. First $\tau_{3} \mid \Sigma=$ inclusion and $\tau_{3} \mid \tau_{2}^{-1}\left(M_{1}\right)=$ $g^{-1} \tau_{2} \mid \tau_{2}^{-1}\left(M_{1}\right)$. Then use Proposition 2.4 to extend $\tau_{3}$ to all of $M^{\prime}$ in such a way that $g \tau_{3}$ is $5 \varepsilon_{1}$-homotopic to $\tau_{2} \operatorname{rel} \Sigma$.

Now define $M^{\prime \prime}=M^{\prime} \times[0,1] /\{\{x\} \times[0,1] \mid x \in \Sigma\}$; i.e., $M^{\prime \prime}$ is obtained from $M^{\prime} \times[0,1]$ by identifying each of the sets $\{x\} \times[0,1], x \in \Sigma$, to a point. Notice that $M^{\prime \prime}$ is $(r+2)$-dimensional and contains two copies of $M^{\prime}: M^{\prime} \times\{0\}$ and $M^{\prime} \times\{1\}$. Roughly speaking, we will attach $M^{\prime \prime}$ to $Z^{\prime}$ along one copy of $M^{\prime}$ to form $Z_{2}$ and shrink out fibers in the other copy to form $Y_{2}$.

The map $\tau_{3}$ defines, in a natural way, a map $\tau: M^{\prime} \times\{0\} \rightarrow Z^{\prime}$. Define $Z^{\prime \prime}=Z^{\prime} \cup_{\tau} M^{\prime}$, define $\xi^{\prime}: Z^{\prime \prime} \rightarrow Z^{\prime}$ to be the map which collapses out the interval factors in $M^{\prime \prime}$ and define $g^{\prime \prime}: Z^{\prime \prime} \rightarrow Z_{1}$ by $g^{\prime \prime}=g \xi^{\prime}$. Then define $Y^{\prime}$ to be the polyhedron obtained from $M^{\prime \prime}$ by shrinking out fibers of the mapping cylinder $M^{\prime} \times\{1\} \subset M^{\prime \prime}$. Let $\mu: Z^{\prime \prime} \rightarrow Y^{\prime}$ be the natural collapsing map.

We now claim that there is a map $\pi: Y^{\prime} \rightarrow P_{1}$ such that $q \pi \mu$ is $8 \alpha_{1}$-homotopic to $\mu_{1} g^{\prime \prime}$ in $Y_{1}$. Since the point preimages of $g^{\prime}$ are precisely the subsets of $Z^{\prime}$ which are identified by $\mu$, we can use $g^{\prime}$ to define $\pi \mid Z^{\prime}$. We can further extend $\pi$ to $Z^{\prime} \cup M^{\prime} \times\{1\}$ by making $\pi(x, 1)=\xi(x)$ for each $x \in M^{\prime}$. In order to extend $\pi$ to the rest of $M^{\prime \prime}$ we must find an $8 \alpha_{1}$-homotopy of $g^{\prime} \tau_{3}$ to $\xi$ which is rel $\Sigma$.

First, there is an $\alpha_{1}$-homotopy of $g^{\prime} \tau_{3}$ to $\mu_{1} g \tau_{3}$ (not rel $\Sigma$ ). Next, there is a $5 \alpha_{1}$-homotopy (this one rel $\Sigma$ ) of $\mu_{1} g \tau_{3}$ to $\mu_{1} \tau_{2}$. But then there is an $\alpha_{1}$-homotopy (rel $\Sigma$ ) to $q \tau_{1}$. Finally, $q \tau_{1}$ is $\alpha_{1}$-homotopic to $\xi$. All of this gives us an $8 \alpha_{1}$-homotopy from $g^{\prime} \tau_{3}$ to $\xi$. On $\Sigma$ this homotopy is not constant, but is the homotopy from $g^{\prime} \mid \Sigma$ to $\mu_{1} g \mid \sum$ followed by the reverse of that same homotopy. We can use a small collar on $\Sigma$ in $M^{\prime}$ to taper off that homotopy and arrive at the homotopy we seek which is rel $\Sigma$. Thus $\pi: Y^{\prime} \rightarrow P_{1}$ is defined. We may assume that $\pi$ is PL and in general position. 
Notice that $q \pi \mu: Z^{\prime \prime} \rightarrow Y_{1}$ and $\mu_{1} g^{\prime \prime}: Z^{\prime \prime} \rightarrow Y_{1}$ are $8 \alpha_{1}$-homotopic. We can also define a CE map $\lambda: Z^{\prime \prime} \rightarrow X_{2}$ by $\lambda=\lambda^{\prime} \xi^{\prime}$. Then the two maps $\lambda_{1} g^{\prime \prime}: Z^{\prime \prime} \rightarrow X_{1}$ and $f \lambda: Z^{\prime \prime} \rightarrow X_{1}$ are $4 \varepsilon_{1}$-homotopic in $X_{1}$.

Special case: $k<(2 / 3)(n-1)$. In this case the dimension of the singular set of $\pi$ is negative and thus $\pi$ is an embedding. We take $Z_{2}=Z^{\prime \prime}, g=g^{\prime \prime}, Y_{2}=\pi\left(Y^{\prime}\right)$, $\mu_{2}=\pi \mu, \lambda_{2}=\lambda$, and $h=q \mid Y_{2}$. Since $\mu_{2}\left|X_{2}=g^{\prime}\right| X_{2}$, we can easily make sure that conclusion (4.19) is satisfied and the proof is complete in this case.

General case. In general, the dimension of the singular set of $\pi$ is less than or equal to $r-1$. We inductively apply the same construction to $\mu \pi$ as was applied above to $g^{\prime}$. This gives new polyhedra $Z^{\prime \prime \prime}$ and $Y^{\prime \prime}$ and a map $\pi^{\prime}: Y^{\prime \prime} \rightarrow P_{1}$. This time we will only have to attach polyhedra of dimension $(r-1)+2=r+1$, and so the dimension of the singular set of $\pi^{\prime}$ will be only $r-2$. This is repeated inductively until $\pi$ is an embedding, which will take at most $r$ repetitions.

5. Proof of Theorem 1. In this section we use the lemmas from the preceding three sections to prove Theorem 1 . The construction is based on that in the proof of Theorem 19 in [Hs].

Let $X, k, r, f, M$, and $n$ be as in the statement of Theorem 1. We begin by embedding $X$ as a tame subset of $\mathbf{R}^{2 k+3}$. The map $f: X \rightarrow M$ can be extended to a compact neighborhood $U$ of $X$ in $\mathbf{R}^{2 k+3}$. We may assume that $f: U \rightarrow M$ is PL and in general position. Fix a sequence $\left\{\boldsymbol{\alpha}_{i}\right\}$ of positive numbers such that $\sum \boldsymbol{\alpha}_{i}<\infty$. Next choose a number $\varepsilon_{1}>0$ such that if $A$ is a compact subset of $U$ and $\operatorname{diam}[A]<\varepsilon_{1}$, then $\operatorname{diam}[f(A)]<\alpha_{1}$. Now let $N_{1}$ be a strong $\left(\varepsilon_{1}, r\right)$-deformation neighborhood of $X$ in $U$. Let us say that $N_{1} \varepsilon_{1}$-collapses to the $k$-dimensional polyhedron $X_{1}$ and that the collapse induces the $\varepsilon_{1}$-retraction $\rho_{1}: N_{1} \rightarrow X_{1}$.

Since $N_{1}$ is a strong $\left(\varepsilon_{1}, r\right)$-deformation neighborhood of $X, f \mid X_{1}: X_{1} \rightarrow M$ is $(r+1)$-connected. We can therefore apply Stallings' construction [St] to $f \mid X_{1}$. This gives us two $k$-dimensional polyhedra $Z_{1} \supset X_{1}$ and $Y_{1} \subset M$, a PL CE retraction $\lambda_{1}$ : $Z_{1} \rightarrow X_{1}$ and a PL CE map $\mu_{1}: Z_{1} \rightarrow Y_{1}$. Furthermore, $Z_{1}$ consists of $X_{1}$ with some mapping cylinders attached. In particular, we can write $Z_{1}=X_{1} \cup M_{1}$, where $M_{1}$ is $(r+2)$-dimensional, $M_{1} \cap X_{1}$ is $(r+1)$-dimensional, and $M_{1} \cap X_{1}$ is collared in $M_{1}$. We can also arrange that $\mu_{1} \mid X_{1}$ is arbitrarily close to $f \mid X_{1}$, and so we choose $\mu_{1} \mid X_{1}$ to be so close to $f \mid X_{1}$ that if $A \subset X_{1}$ and $\operatorname{diam}[A]<\varepsilon_{1}$, then $\operatorname{diam}\left[\mu_{1}(A)\right]<\alpha_{1}$.

We will next apply Lemma 4.1, but we must first specify the number $\varepsilon_{2}$ of the lemma. Choose $\varepsilon_{2}>0$ such that if $A \subset N_{1}$ and $\operatorname{diam}[A]<\varepsilon_{2}$, then $\operatorname{diam}\left[\mu_{1} \rho_{1}(A)\right]$ $<\alpha_{2}$. Now apply Lemma 4.1. This gives us a new diagram

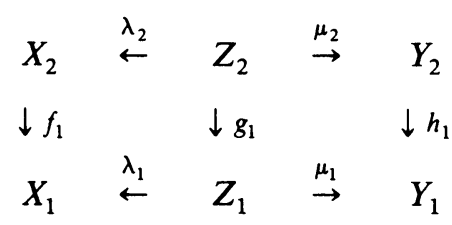

as well as an $\varepsilon_{2}$-neighborhood $N_{2}$ of $X_{2}$. 
Let $\varepsilon_{3}>0$ be a number such that if $A \subset N_{2}$ and $\operatorname{diam}[A]<\varepsilon_{3}$, then $\operatorname{diam}\left[\mu_{2} \rho_{2}(A)\right]<\alpha_{3}$. Apply Lemma 4.1 again, this time to $X_{2}, Z_{2}$, and $Y_{2}$. This procedure is continued inductively and produces an infinite diagram:

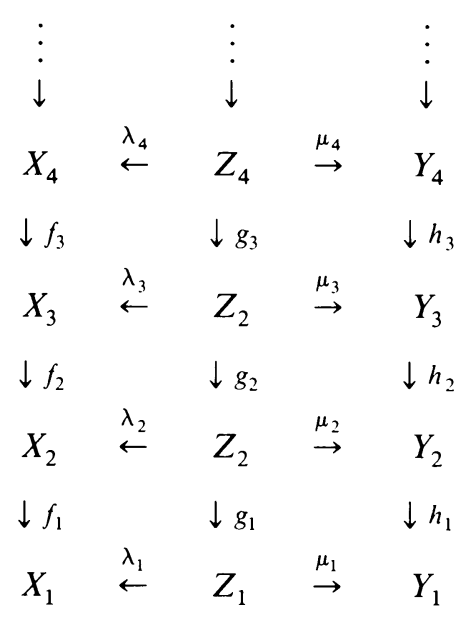

We can make sure that the $\varepsilon_{i}$ are chosen in such a way that $\sum \varepsilon_{i}<\infty$. Let $Z=\lim \left\{Z_{i}, g_{i}\right\}$ and let $Y=\cap P_{i}$. By Proposition 3.2 there are CE maps $\lambda: Z \rightarrow X$ and $\mu: Z \rightarrow Y$. This completes the construction of $Y$. To finish the proof of the theorem we need only show that $Y$ is $\mathrm{LC}^{r}$. To do so, we prove the following statement:

Suppose $L$ is an $(r+1)$-dimensional polyhedron and $L_{0}$ is a subpolyhedron of $L$. For every map $F_{0}: L_{0} \rightarrow Y_{i+1}$ such that $h_{i} F_{0}$ extends to $F: L \rightarrow Y_{i}$ there exists an extension $F^{\prime}: L \rightarrow Y_{i+1}$ of $F_{0}$ such that $h_{i} F^{\prime}$ is $[5+12(r+1)] \alpha_{i}$-homotopic to $F$ rel $L_{0}$ in $Y_{i}$.

This is really just the statement that Proposition 2.4 holds for the neighborhoods $\left\{P_{i}\right\}$ of $Y$. It is easy to see that this implies that $Y$ is $\mathrm{LC}^{r}$ : just take a small singular $m$-sphere in $Y, m \leqslant r$, homotope it to a point in a small subset of $M$, and then apply the statement above to push that homotopy into $Y$ by pushing it into smaller and smaller neighborhoods of $Y$ with a Cauchy sequence of maps.

We will indicate how to get the extension $F^{\prime}$ whose existence was claimed above. It is constructed by using the CE maps $\lambda_{i}$ and $\mu_{i}$ to make the transition from $Y$ to $X$ and back again. We leave the calculation of the size of the homotopies involved to the reader.

Since $\mu_{i+1}$ is CE, we can lift $F_{0}$ to a map $F_{1}: L_{0} \rightarrow Z_{i+1}$ such that $\mu_{i+1} F_{i}$ is arbitrarily close to $F_{0}$. We choose it to be so close that if we can extend the map $\mu_{i+1} F_{i}$, then we are done. Consider $\mu_{i} g_{i} F_{1}: L_{0} \rightarrow Y_{i}$. This map is $8(r+1) \alpha_{i^{-}}$ homotopic to $h_{i} \mu_{i+1} F_{i}$ which extends to all of $L$ (via $F$ ) and thus there is an extension $F_{2}: L \rightarrow Z_{i}$ of $g_{i} F_{1}$. Now consider $\lambda_{i+1} F_{1}: L_{0} \rightarrow X_{i+1}$. Since $f_{i} \lambda_{i+1} F_{1}$ is $4 \varepsilon_{1}$-homotopic to $\lambda_{i} g_{i} F_{1}$ and $\lambda_{i} g_{i} F_{1}$ extends to $\lambda_{i} F_{2}$, we have that $f_{i} \lambda_{i+1} F_{1}$ extends to $F_{3}: L \rightarrow X_{i}$. Thus we are in a position to apply Proposition 2.4 to the maps $\lambda_{i+1} F_{1}: L_{0} \rightarrow X_{i+1}$ and $F_{3}: L \rightarrow X_{i}$ which extends $f_{i} \lambda_{i+1} F_{1}$. Thus there is an 
extension $F_{4}: L \rightarrow X_{i+1}$ of $\lambda_{i+1} F_{1}$ to all of $L$. Now use the fact that $\lambda_{i+1}$ is CE to lift $F_{4}$ to a map $F_{5}: L \rightarrow Z_{i+1}$ which extends $F_{1}$. Now $\mu_{i+1} F_{5}$ is the desired extension of $\mu_{i+1} F_{1}$.

REMARK 5.1. Notice that (in the proof above) $\lim _{\leftarrow}\left\{X_{i}, f_{i}\right\} \subset \lim \left\{Z_{i}, g_{i}\right\}$. So if we did the extra work necessary to make the map $G$ of Lemma 3.1 a homeomorphism, we would have $X \subset Z$ and $\lambda: Z \rightarrow X$ would be a retraction.

REMARK 5.2. It might seem more natural to assume in the statement of Theorem 1 that $f$ is $(r+1)$-shape connected rather than $(r+1)$-connected. But the two assumptions are equivalent because either way we get that the extended map $f$ has the property that $f \mid X_{i}: X_{i} \rightarrow M$ is $(r+1)$-connected (cf. [Hs, Proposition 14]).

\section{REFERENCES}

[Br] M. Brown, Some applications of an approximation theorem for inverse limits, Proc. Amer. Math. Soc. 11 (1960), 478-483.

[Ch] T. A. Chapman, Cell-like mappings, Algebraic and Geometrical Methods in Topology, Lecture Notes in Math., vol. 428, Springer-Verlag, New York, 1974, pp. 230-240.

[C-I] D. Coram and I. Ivanšić, Shape embedding theorem in (co) dimension two, preprint.

[D-H] P. Duvall and L. S. Husch, A continuum of dimension $n$ which does not embed up to shape in $2 n$-space, Proc. Internat. Conf. on Geometric Topology, Polish Scientific Publishers, Warsaw, 1980, pp. 113-119.

[D-S] J. Dydak and J. Segal, Shape theory: An introduction, Lecture Notes in Math., vol. 688, Springer-Verlag, New York, 1978.

[Ed] R. D. Edwards, Demension theory. I, Geometic Topology, Lecture Notes in Math., vol. 438, Springer-Verlag, New York, 1975, pp. 195-211.

[Fe1] S. Ferry, A stable converse to the Vietoris-Smale theorem with applications to shape theory, Trans. Amer. Math. Soc. 261 (1980), 369-386.

[Fe2] , Homotopy, simple homotopy and compacta. Topology 19 (1980), 101-110.

[Hd] J. F. P. Hudson, Piecewise linear embeddings, Ann. of Math. (2) 85 (1967), 1-31.

[Hs] L. S. Husch, $U V^{k}$ maps and embeddings, Topology Appl. 15 (1983), 273-291.

[H-I] L. S. Husch and I. Ivanšić, Embedding compacta up to shape, Shape Theory and Geometric Topology, Lecture Notes in Math., vol. 870, Springer-Verlag, New York, 1981, pp. 119-134.

[Ir] M. C. Irwin, Embeddings of polyhedral manifolds, Ann. of Math. (2) 82 (1965), 1-14.

[La] R. C. Lacher, Cell-like mappings. I, Pacific J. Math. 30 (1969), 717-731.

[L-V] V. T. Liem and G. A. Venema, Stable shape concordance implies homeomorphic complements, Fund. Math. (to appear).

[St] J. Stallings, The embedding of homotopy types into manifolds, mimeographed notes, Princeton University, 1965.

[ک̌́t] M. A. Štańko, The embedding of compacta in Euclidean space, Mat. Sb. 83 (125) (1970), 234-255.

[Wh] H. Whitney, The self-intersections of a smooth n-manifold in 2 -space, Ann. of Math. (2) 43 (1944), $220-246$.

Department of Mathematics, University of Tennessee, Knoxville, Tennessee 37996-1300

Department of Mathematics, Calvin College, Grand Rapids, Michigan 49506 (Current address) 